\title{
Miíase oral em paciente portadora da doença de Wilson: relato de caso
}

\author{
Oral myiasis in patient carrier of wilson's disease: case report
}

Juliana Maria Araújo Silva*

Juliana Andrade Cardoso**

Jener Gonçalves de Farias ${ }^{* * *}$

Antonio Varela Cancio ${ }^{* * * *}$

\section{Resumo}

Objetivo: este trabalho tem como objetivo relatar um caso clínico de míase, acometendo cavidade bucal de paciente portadora de síndrome de Wilson e discutir as principais medidas para a prevenção e o tratamento da mirase. Relato de caso: paciente do gênero feminino, 30 anos de idade, portadora da doença de Wilson, dependente para as atividades da vida diária, apresentando ausência de selamento labial e cuidado de higiene oral inadequado. O diagnóstico de miíase foi estabelecido clinicamente quando observou-se sangramento na boca, presença de larvas e ausência da mucosa do palato. Foi solicitada a internação no centro cirúrgico para debridamento do tecido e remoção das larvas. Durante procedimento ficou constatado presença de colônia de larvas também no interior da língua. Considerações finais: a ocorrência de miíase em cavidade bucal é rara. Alguns fatores são predisponentes, dentre esses: a senilidade, as doenças e debilidades neurológicas, a halitose. O principal tratamento constitui-se de remoção mecânica e prescrição medicamentosa antibacteriana e antiparasitária. A paciente do caso em análise foi tratada em centro cirúrgico, com remoção mecânica das larvas, e os responsáveis por ela foram orientados para uma higiene oral adequada para evitar a reinfestação.

Palavras-chave: Miíase. Cavidade oral. Língua. Palato. Doença de Wilson.

\section{Introdução}

O termo "miíase" é derivado do grego myio (mosca) e ase (doença) e é usado para definir a invasão dos tecidos vivos humanos e de outros mamíferos por ovos ou larvas de moscas da ordem díptera, sendo mais frequentemente observada em países tropicais ${ }^{1}$. As larvas de Cochliomyia hominivorax e Dermatobia são os agentes causais mais comuns das miíases humanas na América. A ocorrência de miíase bucal é rara e há poucos relatos de casos clínicos na literatura descrevendo essa condição $0^{2,3}$.

As miíases são classificadas de acordo com os aspectos biológicos e evolutivos. Dividem-se em dois grandes grupos: biontófagas e necrobiontófogas. As biontófagas ou parasitárias, são caracterizadas como invasores primários, tem a capacidade de invadir tecidos sadios e feridas recentes. Já, as necrobiontógafas ou facultativas, nutrem-se de tecidos orgânicos em decomposição ou podem atingir eventualmente tecidos necrosados em um hospedeiro vivo ${ }^{1,4}$.

As manifestações clínicas não são específicas e variam de acordo com a região acometida ${ }^{5}$. A ocorrência de míase em cavidade bucal é rara e, nesses casos, a localização mais frequente é a região anterior de maxila. $\mathrm{O}$ aspecto pode variar de pequenas

Cirurgiã-dentista pela União Metropolitana de Educação e Cultura (Unime), Lauro de Freitas, Bahia, BA, Brasil.

Professora do Núcleo de Propedêuticas Clínica e Cirúrgica da União Metropolitana de Educação e Cultura (Unime), Lauro de Freitas, BA; Mestra em Estomatologia Clínica pela Pontifícia Universidade Católica do Rio Grande do Sul (PUCRS), Porto Alegre, RS, Brasil; Especialista em Estomatologia pela União Metropolitana de Educação e Cultura (Unime), Lauro de Freitas, Bahia, BA, Brasil.

* Professor adjunto de Cirurgia e Traumatologia Bucomaxilofacial (CTBMF) da Universidade Estadual de Feira de Santana (UEFS), Feira de Santana, Bahia, BA; professor do Núcleo de Propedêuticas Clínica e Cirúrgica da União Metropolitana de Educação e Cultura (Unime), Lauro de Freitas, Bahia, BA, Brasil; Doutor em Estomatologia pela UFPB; Mestre em Cirurgia e Traumatologia Bucomaxilofacial pela FOP-UPE.

**** Professor substituto de Cirurgia e Traumatologia Bucomaxilofacial (CTBMF) da Universidade Estadual de Feira de Santana (UEFS), Feira de Santana, BA, Brasil; Mestre em Odontologia pela Universidade Federal da Bahia (UFBA), Bahia, BA, Brasil; Especialista em Cirurgia e Traumatologia Bucomaxilofacial (CTBMF) pela Universidade de Pernambuco (UPE), Pernambuco, PE, Brasil. 
úlceras a grandes destruições teciduais com a presença de larvas e sua constatação é a chave para o diagnóstico ${ }^{6}$.

De acordo com a localização e o tecido envolvido há diferentes nomenclaturas, como: miíase gengival, miíase dental, miíase periodontal e miíase oral ${ }^{1}$. Alguns fatores são predisponentes, como higiene bucal deficiente, falta de selamento dos lábios, resistência corporal diminuída, desnutrição, respiração bucal, etilismo, senilidade, comprometimento neurológico, hemiplegia e traumas na área facial $^{4,7}$. Dessa forma, pacientes com necessidades especiais tornam-se mais vulneráveis.

A síndrome de Wilson é uma doença rara, sendo classificada como uma pseudoesclerose. Em 1912, foi descrita sua forma clássica, correlacionando a sintomatologia dos gânglios da base à doença do fígado, denominando-se degeneração hepatolenticular. Essa degeneração origina-se de uma transmissão autossômica recessiva, que provoca alteração no metabolismo do cobre, comprometendo o metabolismo de uma enzima, ceruloplasmina e depositando cobre em alguns órgãos principalmente no fígado, no cérebro e na córnea. $O$ excesso de cobre danifica os órgãos afetados. Essa doença acomete indivíduos de 11 anos a 25 anos, causando problemas neurológicos, hepáticos, psiquiátricos e oculares ${ }^{8}$.

O principal tratamento para a miíase constitui-se de remoção mecânica e prescrição medicamentosa antibacteriana e antiparasitária ${ }^{2}$.

O presente trabalho objetiva relatar um caso clínico sobre mííase bucal em paciente portadora da doença de Wilson, com debilidade neurológica, e discutir as medidas possíveis para prevenção e para o tratamento, de modo a orientar profissionais da saúde e cuidadores sobre a relevância dos cuidados com a saúde bucal.

\section{Relato de caso}

Paciente do gênero feminino, leucoderma, solteira, 30 anos de idade, portadora da doença de Wilson, totalmente dependende para as atividades da vida diária. Apresentou-se ao Hospital Clériston Andrade, em Feira de Santana-Bahia (Ba), trazida por sua cuidadora, solicitando avaliação bucomaxilofacial após ser observado sangramento, secreção e odor fétido em cavidade oral. Ao exame clínico, observou-se uma grande quantidade de larvas na região do palato duro, do palato mole e da língua (Figuras 1 e 2).

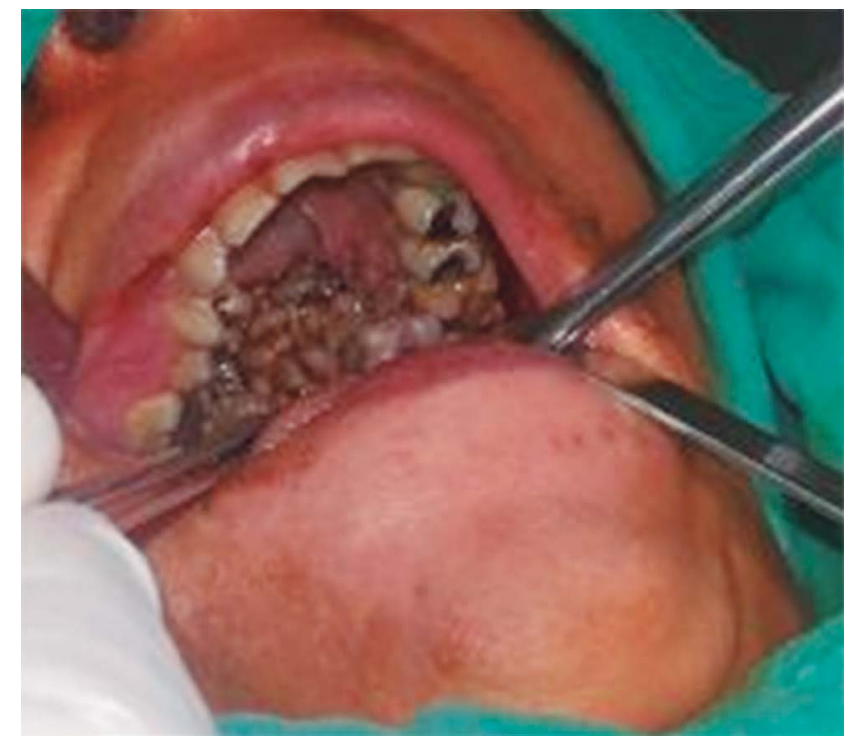

Fonte: dos autores.

Figura 1 - Remoção mecânica das larvas de mosca e debridamento do tecido do palato

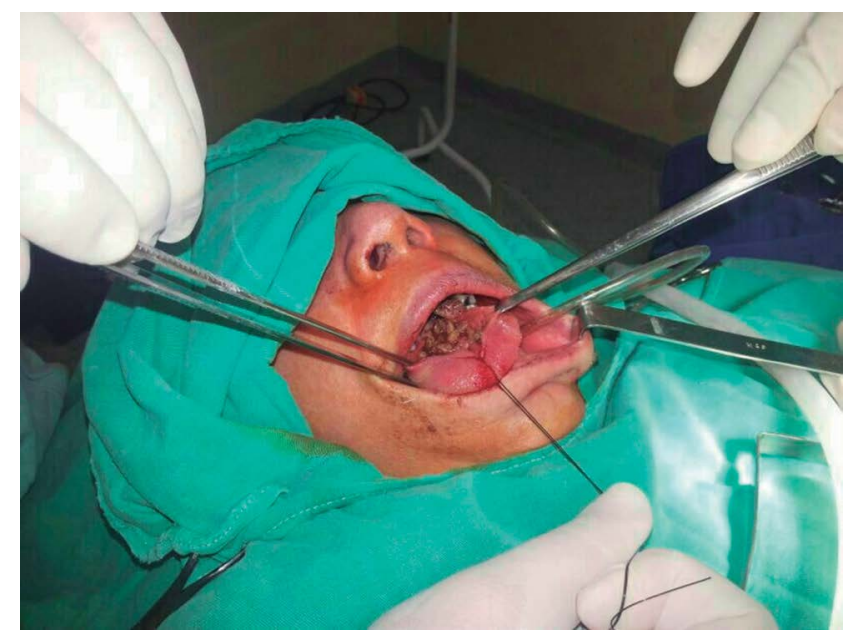

Fonte: dos autores.

Figura 2 - Remoção mecânica de grande quantidade de larvas de mosca no interior da língua

Após exame físico, foi dado o diagnóstico de miíase bucal. A conduta adotada foi: internamento hospitalar; solicitação de traqueostomia; admissão em centro cirúrgico; anestesia geral para remoção completa das larvas, com média de mil larvas, em diversos tamanhos; e debridamento de tecido mole afetado. Todo o tecido que recobria o palato duro foi completamente removido, expondo-se todo o osso. A ferida na língua e no palato foi deixada para fechamento em uma segunda intenção (Figura 3). 


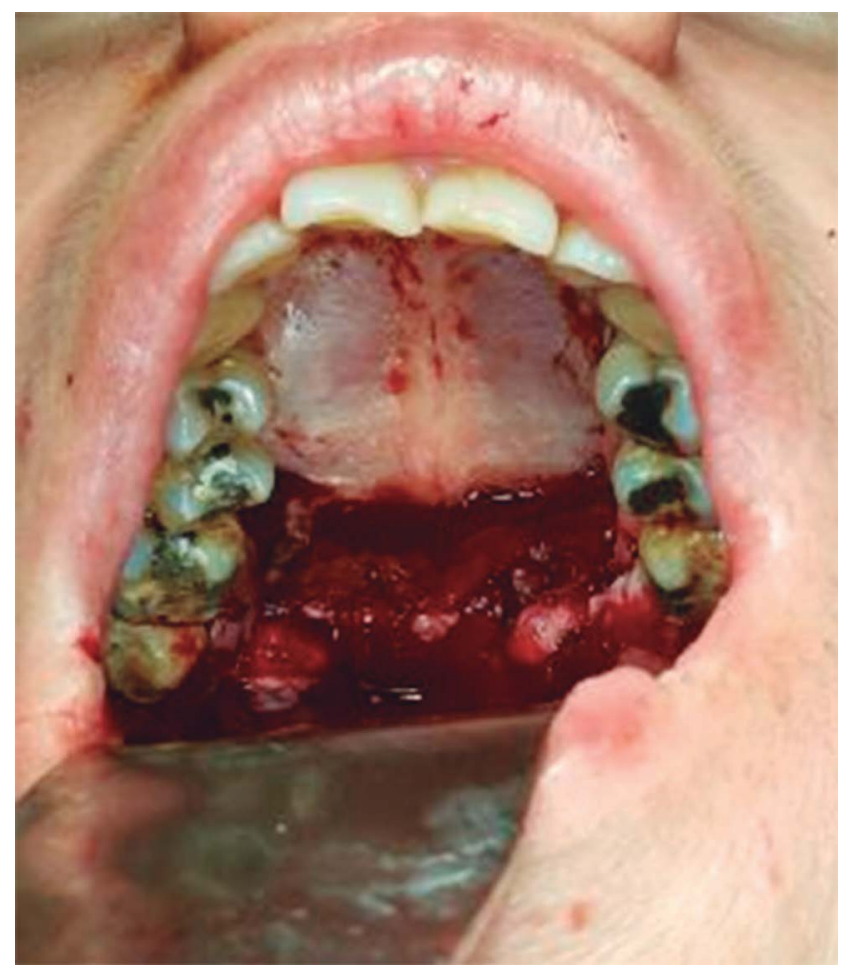

Fonte: dos autores.

Figura 3 - Vista do palato após remoção mecânica das larvas e do debridamento

A conduta pós-operatória foi a prescrição de antibiótico, anti-inflamatório e analgésico (dexametasona $10 \mathrm{mg}$, cetoprofeno $100 \mathrm{mg}$, metronidazol 500 $\mathrm{mg}$, cefalotina $1 \mathrm{~g}$ e dipirona sódica $1 \mathrm{~g}$, todos sendo ingeridos por sete dias). Os cuidadores foram orientados quanto ao uso de máscara no paciente, devido à ausência de seu selamento labial e em relação à melhoria da higiene oral.

Após trinta dias, a paciente retornou para a reavaliação. Foi possível observar discreta melhora na higiene bucal e boa cicatrização, com formação de nova mucosa palatina, sem sinais de infecção por larvas.

\section{Discussão}

A miíase corresponde a uma infestação de tecidos vivos pelas larvas de moscas.

Essas moscas aparecem em maior quantida$\mathrm{de}^{2,9,10}$ principalmente em países tropicais, de clima quente e úmido, nos meses chuvosos de verão. O Brasil é uma região propícia ao desenvolvimento das principais espécies de moscas que causam miíase em humanos, o que favorece o desenvolvimento dessa afecção $0^{2,11}$.

A ocorrência bucal da miíase é rara ${ }^{2,3}$ e torna-se mais comum em pessoas idosas, debilitadas, doentes e com deficiência mental ${ }^{11}$. No presente caso, a paciente em questão era moradora de uma cidade do nordeste brasileiro, portadora da síndrome de Wilson, uma doença rara que provoca alteração no metabolismo do cobre.
Essa doença acomete indivíduos de 11 anos a 25 $\operatorname{anos}^{8}$ e, $40 \%$ a $60 \%$ dos pacientes com a Síndrome de Wilson apresentam suas primeiras manifestações neurológicas, acometendo o sistema nervoso central, predominantemente com distúrbios motores, tais como tremor, rigidez, distonia, coreia e ataxia. Dessa forma, o paciente portador dessa síndrome torna-se dependente de cuidadores e familiares ${ }^{12}$. A paciente em questão com 30 anos de idade era totalmente dependente dos seus cuidadores, corroborando as informações da literatura.

Os profissionais envolvidos no cuidado dos pacientes com necessidades especiais de cuidados de saúde devem conhecer estratégias para prevenção, diagnóstico e tratamento de tal doença. A miíase bucal, ainda que mais rara, é descrita em pacientes com higiene bucal deficiente, respiração bucal, falta de selamento dos lábios e halitose severa. Esses fatores atraem as moscas para deposição de larvas ${ }^{3,11}$.

A avaliação da cavidade bucal de indivíduos portadores de cuidados especiais de saúde é a principal estratégia para a prevenção da infestação por larvas de moscas e outras patologias bucais. Além disso, adoção de medidas de proteção individual e coletiva como telar portas e janelas de hospitais ou dependências domésticas, evitar exposição de feridas abertas, ulcerações com tecido necrosado, eczemas infectados, assim como manter boa higiene individual e ambiental, e adotar o uso de máscaras em pacientes com ausência de selamento labial são procedimentos de suma importância para se prevenir a instalação de miíase ${ }^{11}$.

A ausência de selamento labial e a total dependência por parte da paciente, associada à falta de informação da cuidadora sobre as medidas preventivas para se evitar o aparecimento da miíase contribuíram para a instalação das larvas no presente caso. Além disso, as condições de higiene eram precárias, o que levou ao agravamento da doença.

A chave para o diagnóstico de miíase é a constatação da presença das larvas ${ }^{6}$. O tratamento de escolha de acordo com os estudos revisados é a realização da remoção mecânica das larvas, porém, em casos mais severos a antibioticoterapia sistêmica deve ser adotada ${ }^{11}$, estando, portanto, o caso relatado de acordo com a literatura.

\section{Considerações finais}

Pacientes portadores de necessidades especiais de saúde, dentre eles, pacientes com a doença de Wilson, constituem um grupo de risco para desenvolvimento de miíase bucal. A prevenção dessa patologia constitui um desafio para cuidadores e familiares, especialmente no caso de pacientes com ausência de selamento labial. A informação e a orientação para familiares, cuidadores e para as instituições de longa permanência em relação aos cuidados bucais e ambientais, assim como a avaliação rotineira 
da condição bucal desses pacientes constituem as principais estratégias preventivas para esse grupo. O principal tratamento paliativo para a alteração baseia-se na remoção mecânica das larvas e instituição do uso oral de antibióticos. No presente caso, a paciente foi tratada com remoção mecânica das larvas e medicações sistêmicas. Os responsáveis foram orientados para uma higiene oral adequada, a fim de evitar a reinfestação.

\section{Abstract}

Objective: this paper aims to report a clinical case of myiasis affecting the oral cavity of a patient with Wilson's disease, and to discuss the main steps for prevention and treatment of myiasis. Case report: female patient, 30 years old, carrier of Wilson's disease, dependent on others for daily activities, with no lip closure and inadequate oral hygiene care. The diagnosis of myiasis was clinically established upon observation of mouth bleeding, larvae presence, and absence of the palatal mucosa. Admission to the surgical center was requested for debridement of tissue and larval removal. During the procedure, it was verified the presence of larval colony also inside the tongue. Final considerations: the occurrence of myiasis in the oral cavity is rare. Some factors are predisposing, among them: senility, neurological diseases and shortcomings, and halitosis. The main treatment consists of mechanical removal, and antibacterial and anti-parasitic drug prescription. The patient in the present case was treated in the surgical center with mechanical larval removal, and those responsible for the patient were instructed for proper oral hygiene to prevent reinfestation.

Keywords: Myiasis. Oral Cavity. Tongue. Palate. Wilson's Disease.

\section{Referências}

1. Gomes de Araújo RJ, Hanna LMO, Gomes L, Carvalho LHV. Cochliomyia homnivorax em estágio avançado na cavidade oral. RGO. 2009;57(2):229-33.

2. Cavalcanti AL. Miíase Oral: etiologia, diagnóstico e tratamento. Rev Fac Odontol Porto Alegre 2008; 49(2):32-5.

3. Thyssen PJ, Nassu MP, Costella AMU, Costella ML. Record of oral myiasis by Cochliomyia hominivorax (Diptera: Calliphoridae): case evidencing negligence in the treatment of incapable. Parasitol Res. 2012;111:957-9.

4. Oliveira JAGP, Machado MI, Oliveira MP. Miíase de língua: relato de um caso de infestação hospitalar. Rev Cir Traumatol Buco-Maxilo-fac. 2008; 8(4):47-50.

5. Freitas DA, Moreira G, Jones KM, Caldeira AP. Social exclusion and myiasis of the upper lip in a Brazilian rural resident. Rural and Remote Health [periódico online] 2012 [Citado 2014 Oct. 24]; 12:2188. Disponível em URL: http:// www.rrh.org.au

6. Cencil J, Zardo M, Takahashi A, De Sá ACD, Martins LD, Gonçalves RCG. Publ UEPG Ci Biol Saúde 2006; 12(2):39-43.

7. Martins Junior JC, Keim FS, Iarocrinski J. Oftalmomiíase pós-traumática: relato de caso e revisão de literatura. Ver Bras Oftalmol. 2010; 69(4):264-8.
8. Brito JCF, Coutinho MAP, Almeida HJF, Nóbrega PV. Doença de Wilson: Diagnóstico clinic e sinais das "faces do panda" à ressonância magnética. Arq Neuropsiquiatr. 2005;63(1):176-9.

9. Batt AP, Jayakrishnan A. Oral myiasis: a case report. Int J Paediatr Dent. 2000;10(1):67-70.

10. Linhares AX. Miíases. In: Neves DP, Melo AL, Genaro O, Linardi PM. Parasitologia Humana. 10ed. São Paulo: Ed. Atheneu, 2000. Cap.48, p.350-7.

11. Ribeiro MTF, Sanglard-Oliveira CA, Naves MD, Ferreira EF, Vargas AMD, Abreu MHNG. Miíase bucal e doença de Alzheimer: relato de caso clínico. Rev. bras. geriatr. Gerontol. 2012; 15(4):805-11.

12. Machado AAC. Manifestações neurológicas na doença de Wilson: estudo clínico e correlações genotípicas [Tese de Doutorado]. São Paulo: Faculdade de Medicina da Universidade de São Paulo; 2008.

\section{Endereço para correspondência:}

Juliana Maria Araújo Silva

Rua da Mangueira, 24 - Vila de Abrantes 42840-000 Camaçari-BA

Telefone: (71) 36234123

Recebido: 03/11/2014. Aceito: 05/12/2014. 